\title{
ATE Enabling Technologies: Present and Future
}

\author{
Larry V. Kirkland, OO-ALC/TISA \\ Jeffrey S. Dean, SA-ALC/LDAE
}

\begin{abstract}
A discussion of the current and emerging core technologies and philosophies that will enable Air Force personnel to quickly, accurately and intuitively diagnose faults in increasingly complex systems.
\end{abstract}

\section{INTRODUCTION}

Until recently, ATE has been limited in capability by the hardware and software technology available. Rather than work with our strengths, ATS user interfaces have historically played on our weaknesses. The ATS user, through long training and experience has had to adapt to the machine, rather than the machine adapting to the user. After the user was trained, he often moved on to another job or eventually retired, taking his expertise with him. This would require another long learning period as a new user adapts to the ATS.

With the advent of fast microprocessors and graphical, intuitive software interfaces our computers, and ATE, are beginning to adapt to us. Graphical, object oriented user interfaces, machine-based intelligence and perception, virtual realism, speech recognition and other emerging capabilities are making or will make ATE easier to understand and use. In addition, the push for open hardware and software standards, object oriented programming and software libraries will allow faster ATS and TPS design and generation, providing the flexibility ne eded to ad a p t to changing requirements and technologies.

\section{HUMAN WEAKNESSES AND THE COMPUTER}

The ability to read, perform mathematical analysis, and to logically attack and solve a problem are, unfortunately, not natural functions for the human species. Instead we are trained over much of our childhood (hopefully) to learn to use these skills. Given the current state of American education, too few of our children learn these skills adequately enough to be considered competent. Most of us can on the other hand recognize a friend in a crowd, make a guess based on incomplete information, or create solutions to problems by generalizing from similar problems solved in the past.

Computers, and ATE, have required us to operate in the realm of our weaknesses: details are demanded while we tend to generalize facts, keyboard/textual interaction (often in arcane codes or commands) is required of beings who process data visually (spatial interrelationships) and orally (speech). With time and training, we can acquire the skills needed to work with these machines. Our learned ability to operate in a manner that is not natural and requires more effort is usually referred to as a skill. The more skilled we are considered to be, the less natural our behavior and sensory processing have become. Eventually, after the person obtains the training, skills and experience needed to alter ones behavior to adapt to the computer, the person is moved somewhere else, starting a new cycle of frustration and inefficiency.

We can, as adaptable humans, learn to operate and think in these skill-based, text dependent modes, but there are costs. Training time is required to use any ATE, errors are made that must be corrected, efficiency is lost by having to perform in a non-visual, skill intensive environment. As the Air Force downsizes (or rightsizes), it can afford less and less to pay these costs. We are hemorrhaging our skills base as our senior technicians take early retirement, while training funds are harder to come by for those who remain. Errors in repair become more important as the spares supplies dwindle, and efficiency becomes critical as our workforce gets smaller while the job of the Air Force only gets bigger. 


\section{DRAWING FROM/ COMPLEMENTING HUMAN STRENGTHS}

Under what conditions can we expect a complex dynamics of information to emerge spontaneously and come to dominate human behavior[4]? Human beings are highly visually oriented. We look for patterns, we can "spot" mistakes, and when we understand something it is often acknowledged with "I see!" Someone with exciting new ideas is often described as being a visionary. Our visual orientation is reflected in our brain; a significant chunk of our cerebral cortex is devoted towards extracting, identifying and interrelating objects within our visual space, creating a world view of our surroundings within our mind. Within our internal visual world we can extend ourselves to incorporate phenomena, concepts or objects that are not physically existent, such as Einstein's experiments in the imagination that he used to understand relativity. Essentially we have within us a very flexible, massively parallel visual processor that has been largely unused in the past when trying to diagnose faults within electronic systems via ATE.

We can improve ATE effectiveness by tapping into this visual processing capability in two basic ways. First, by using graphical objects on the ATS monitor we can display the components on a circuit card as a visual, object oriented representation; by selecting chips, capacitors, resistors, etc. with a mouse we can call up applicable tests, voltage levels, and failure data associated with that object.

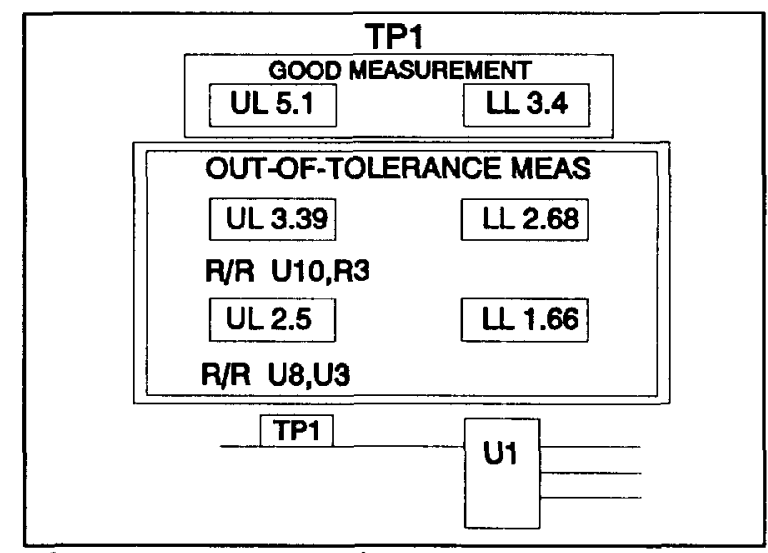

Figure 1. Test Points.

We can navigate through windows, icons, buttons, menus and synthetic test devices to drive the ATS to perform tests, initiate diagnostic algorithms, and display results. In other words we can create a graphical world, a "virtual reality" in which we manage our ATE and manipulate data in an intuitive manner. The appeal of operating in the graphical environment is obvious. Anyone who doubts the benefits and appeal of the graphical interface can try telling the attendees at any software convention how they love DOS, because it is easier to work with than Windows. Possible consequences of this action include being laughed at or being anesthetized and put in a rubber room.

The second way in which we can assist ourselves visually in diagnosing a faulty circuit card is to expand our ability to "see" the card. X-ray technology can allow us to look for broken circuit paths, spot bad or marginal solder joints, or derive interconnections for reverse engineering of a card no longer in production. Infra-red imaging can spotlight chips operating hotter or cooler than normal, indicating the improper functioning of that chip or the components connected to it. The imaging of the electrical fields around the circuit card can show where the current is flowing and where it is not, and whether it is AC or DC (Fig. 2).

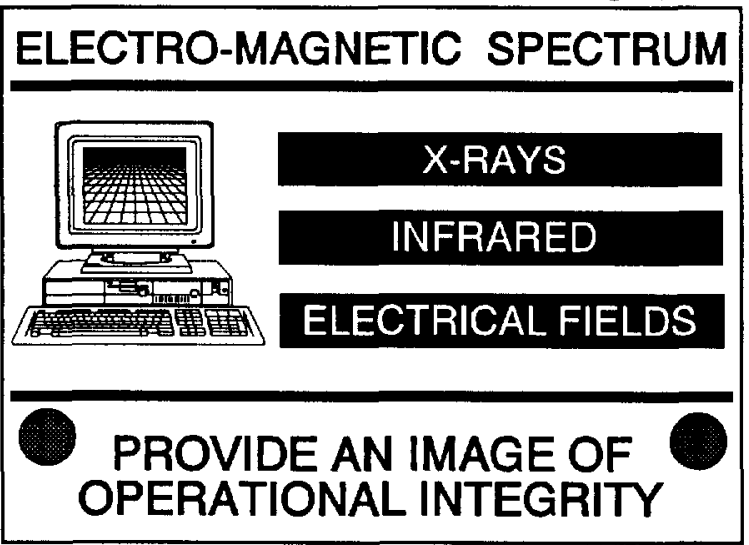

Figure 2. Electro-magnetic.

Because of our ability to extract and compare visual patterns quickly, these methods give us a fast means of determining the location and type of failure involved.

Another information rich sense that humans use naturally is that of hearing, in particular the ability to speak and to understand speech. Being able to talk interactively with a computer has been a dream for decades and, until recently, has been considered science fiction. With the advances in speaker independent speech recognition, this capability may soon become science fact. Such a capability would able an ATS user to operate the ATS with his hands free to manipulate connections to the UUT for various tests. With voice responses the system could provide test results verbally, freeing the user from being trapped at a monitor as tests are run. This freedom to leave the keyboard adds a dimension to the user's capabilities, allowing him to perform other operations in parallel with controlling the ATS. 


\section{KEEPING HUMAN EXPERTISE IN THE MACHINE}

While the above technologies can make computers (and ATS) easier to learn and to operate, the user must still direct the machine to act in accordance with his experience and knowledge. An experienced ATS user knows the failure modes of the circuit cards he tests; he knows which tests fail most often. In possessing this knowledge, the experienced user can accurately diagnose the bad component(s) on a faulty card much faster than the novice, often just by looking at the card (heat discoloration, cold solder joints). Every test organization has its share of wizards and gurus, who understand the idiosyncrasies of a particular ATS or UUT. When such a person leaves, the organization loses capability and money, as it trains someone to replace him.

The answer to this dilemma is to retain expertise within the ATS itself. This can be achieved by incorporating various forms of Artificial Intelligence in the ATS, with the ability to extract failure information from each UUT tested and to learn from them. At first, any such machine would require some baseline knowledge about the UUT and its failure modes, drawn from the UUT design and specifications. This is necessary for the initial testing of the UUT in order to have some hope of rendering a correct diagnosis. After the testing is completed and the failed component identified and confirmed, the ATS can begin associating specific test failures, X-ray, infra-red or electromagnetic field image patterns, or signal strength levels with different failure modes. Each time that type UUT is tested, the ATS becomes smarter in identifying the failed components.

\section{MACHINE-BASED INTELLIGENCE}

Machine-based Intelligence can be viewed as Adaptive Information Processing Systems designed to alter its response to specific criteria or events which results in optimizing task quality and performance. Advanced emergent concepts including Neural Networks, Genetic Algorithms, Cellular Automata, Lindenmayer Systems, etc., can be used to effectively generate, maintain, and optimize operational processes. Machine-based Intelligence can be used on ATE to control the TPS testing sequence, to provide alternative, high-speed accurate fault-diagnostics and to provide advice as to the optimal testing technology.

\section{RETARDUS ILLUMINOUS GIGANTUS (Slow Blinking Monsters)}

ATE has been technology limited. Depending on the application, to integrate all of the stimulus and measurement devices for a specific application could have required a test system 50 feet long or more. The computing power of the system was also technology limited and most definitely a closed system. ATE is evolving as technology evolves. Our task is to stay in line with the technology and work together as a united technology team, both industry and government, in an open architecture environment. An open-architecture hardware environment is a platform in which stimulus and measurement modules can be intermixed using equipment from different manufacturers to make up a system. An open-architecture software environment is a platform in which different software packages can be integrated into an interoperable system.

\section{OPEN ARCHITECTURE}

Off-the-shelf operating systems (i.e., DOS, UNIX, WINDOWS, etc.) provide a high performance platform for machine intelligent implementation on ATE. Personal computers provide versatility with their high speed, memory manageable, open systems. The IBM 486 AT architecture has more processing power than many of the large, multi-cabinet ATS packages in use today, and with it's universal availability, upgradability and price, it is surprising that everyone has not jumped on the bandwagon of hosting new or upgraded TPSs on this platform. In the near future, small businesses may very well be undercutting major ATS suppliers by offering the same functionality at less than a tenth of the cost, simply by taking advantage of the power of this architecture.

Open architectures in an integrated technology environment permit the combination of multiple information sources for the diagnostic evaluation of a UUT. Open systems provide interactivity, interoperability, multiple languages, graphics, multiple technology and a pick and choose testing platform. The Air Force currently has far too much proprietary ATS purchased to support specific weapon systems, which over time have become unsupportable and expensive, with no clear upgrade path.

VXI testing platforms generally are open, modular, compact and easier to integrate than any technology in existence today (1). VXI has inspired new approaches to building modular instrument systems, and it has also allowed advanced approaches to integrating these components with software to build system level open solutions. It is crucial that VXI remain an open standard, rather than have various proprietary spin offs that are incompatible, and cannot communicate or talk to each other. The Air Force is looking at long term, life cycle costs for new ATS acquisitions, and closed systems are more expensive in the long run to manage and upgrade. Figure 3 shows a typical VXI layout. 


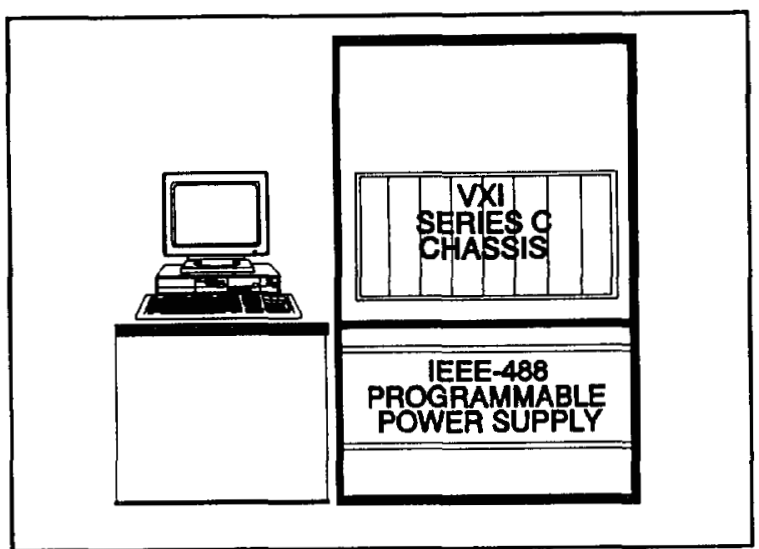

Figure 3. VXI Tester.

\section{INTEROPERABILITY STANDARDS}

No standard exists at this time for the expression of information and knowledge content at the domain level for specific applications of Knowledge-based learning systems. Critical issues are therefore:

- How do we talk back and forth (how do programs communicate)

- How do we structure our sentences so we can understand one another (what are we conveying)

-What should be the specific domain level words or expressions.

An activity to develop a standard ontology of common terms to represent sensor and sensor-based signals in Knowledge-based learning Systems is in work (2). Ontology provides a lexicon of common terms, providing the users with a common dictionary of standard labels and units which have a precise, well-defined meaning, independent of the internal or individual processing system. The Sensor-based Ontology is an ATE-specific ontology which provides for interoperability between different software packages, testers, sensors, and Instrument on A Card (IAC). Interoperability is talking the same language, using the same terms, and inferring the same meaning; so no matter what internal or individual system processing is used at least the conveyance of sensor-based information will be understood. Essentially, interoperability is the ability to communicate between software packages and to understand what is said. A simulation of potential information exchange is shown in Figure 4.

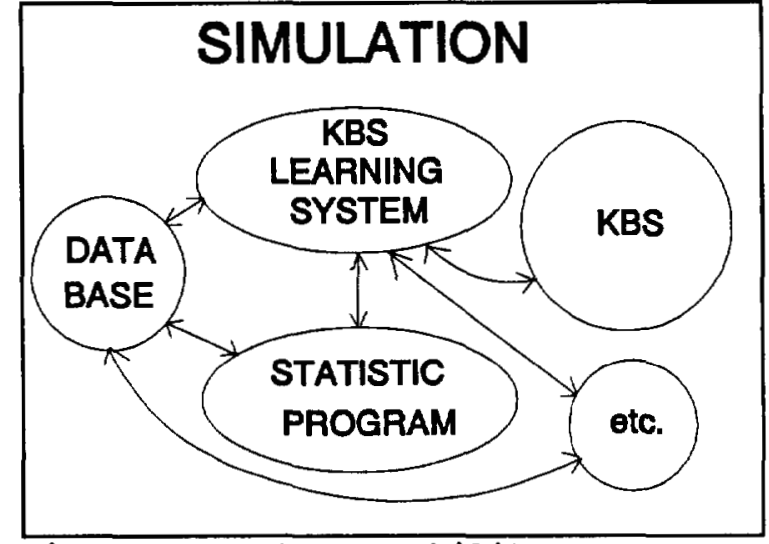

Figure 4. Interoperability.

\section{ABET}

There are currently several flavors of ATLAS in use on different ATS platforms, which makes rehosting a TPS to a different test platform difficult, time consuming and expensive. While ATLAS is the most common language to be found in ATS, it is not the only one. Because of this plethora of languages and language variants, it is the software costs (development and maintenance) that usually drive the life cycle costs of any of the more complex ATS.

ABET is a set of software interface standards for the test domain to facilitate the transition of test information and implementation of automated tests (3). These software interface standards are defined to support software component portability, reusability, and exchangeability and to serve as targets for test-related development tools. ABET holds the potential for greatly simplifying TPS development with its emphasis on modular, object oriented programming, software reuse, and standardization of software/software and hardware/software interfaces.

\section{CONCLUSIONS}

One of the major limitations of testing electronic components is that it requires a logical, knowledge intensive approach. This is a limitation because people think in an intuitive, vertical manner. Rather than playing to our strengths, computers (and ATE) have forced us to operate in the realm of our weaknesses; details are demanded while we tend to generalize facts, keyboard/text interaction is required of beings who process data visually (spatial interrelationships) and orally (speech). Eventually, after the person obtains the training and experience needed to alter their behavior to adapt to the computer, they are moved somewhere else, starting a new cycle of frustration and inefficiency. 
People have a tendency to learn and operate in a multimodal sensory world. Computers have traditionally forced us to rely on textual interchanges, a "limited bandwidth" form of communication. Graphical object oriented interfaces, combined with speech generation for perception, would allow users to interact with computers (and ATE) on multiple levels.

Not only should the ATE adapt to our ability to fuse various sensory inputs (i.e., visual and auditory) to create a better interpretation of the available data, but it should also be able to perceive and integrate information beyond human sensory abilities. Thermal and X-Ray imaging, electromagnetic field detection of the unit under test provide valuable clues to the ATE as to the cause of failure. The fusion and interpretation of extended sensory information into the ATE augments system accuracy, and usability, particularly for users with less experience. An extension of this graphical interface to a "virtual reality" could complete the transition from a totally logical, "leftbrain" approach of problem solving to one of a balanced, "whole-brain" approach, blending logic with our ability to derive solutions.

Adaptive Machine-based Intelligence (Neural Networks, algorithms) systems coupled with automated learning can help the user track and utilize all available data needed for decision making, and can emulate a human's ability to extrapolate from the partial data available to reach conclusions. Because the expertise in diagnosing faults in a system resides largely within the test equipment, turnover of trained personnel does not significantly impact mission effectiveness.

Finally, we the Air Force depend on thousands of special purpose, unique pieces of test equipment, in which we cultivate "experts" to use and maintain. When the experts leave, the system fails and our mission is impacted. To counter this, we must move away from those factors which create specialized ATE systems; proprietary hardware, software and data structures that impede easy maintenance and modifications, and steepen the user learning curve. Standardized and open hardware and software interfaces, plus common data formats including inter-operability make it possible to mix and match equipment sets to meet testing requirements. In particular, the current power of the PC (486) will make it possible to replace large, expensive, proprietary ATS with equivalent or greater functionality in the near future.

The Air Force is looking at the long range costs of new ATS, including training requirements, effectiveness, and upgradability. These costs can be lowered by the acquisition of systems that are intuitive to use, intelligent, with open software and hardware for easy maintenance and upgrading. With the do more with less philosophy now in place, we can not afford to do otherwise.

\section{REFERENCES:}

1. Ron Wolfe, EE Engineering Evaluation, "The Status of VXI", April 1993.

2. R. Glenn Wright, ARPA/GMA Industries - KnowledgeBased system interoperability for Sensor-Based Processing Applications.

3. ABET, Proposed Draft IEEE Trial-Use Standard, 1992.

4. Christopher G. Langton, Charles Taylor, J. Doyne Farmer, Steen Rasmussen, "Artificial Life II" Feb 1990. 